\title{
NuSTAR Hard X-ray Optics Design and Performance
}

\author{
Jason E. Koglin ${ }^{a^{*}}$, HongJun An ${ }^{a}$, Kenneth L. Blaedel ${ }^{\mathrm{a}}$, Nicolai F. Brejnholt ${ }^{\mathrm{b}}$, Finn E. Christensen ${ }^{\mathrm{b}}$, \\ William W. Craig ${ }^{\mathrm{c}}$, Todd A. Decker ${ }^{\mathrm{a}}$, Charles J. Hailey ${ }^{\mathrm{a}}$, Layton C. Hale ${ }^{\mathrm{a}}$, Fiona A. Harrison ${ }^{\mathrm{d}}$, \\ Carsten P. Jensen ${ }^{\mathrm{b}}$, Kristin K. Madsen ${ }^{\mathrm{d}}$, Kaya Mori ${ }^{\mathrm{a}}$, Michael J. Pivovaroff ${ }^{\mathrm{c}}$, Gordon Tajiri ${ }^{\mathrm{a}}$, William \\ W. Zhang ${ }^{\mathrm{e}}$
}

\author{
${ }^{a}$ Columbia Astrophysics Laboratory, New York, NY \\ ${ }^{\mathrm{b}}$ Danish Technical University, Copenhagen, Denmark \\ ${ }^{c}$ Lawrence Livermore National Laboratory, Livermore, CA \\ ${ }^{\mathrm{d}}$ California Institute of Technology, Pasadena, CA \\ ${ }^{\mathrm{e}}$ Goddard Space Flight Center, Greenbelt, MD
}

\begin{abstract}
The Nuclear Spectroscopic Telescope Array (NuSTAR) is a NASA satellite mission scheduled for launch in 2011. Using focusing optics with multilayer coating for enhanced reflectivity of hard X-rays (6-79 keV), NuSTAR will provide a combination of clarity, sensitivity and spectral resolution surpassing the largest observatories in this band by orders of magnitude. This advance will allow NuSTAR to test theories of how heavy elements are born, discover collapsed stars and black holes on all scales and explore the most extreme physical environments. We will present an overview of the NuSTAR optics design and production process and detail the optics performance.
\end{abstract}

Keywords: Hard X-ray telescope, X-ray optics, segmented glass, NuSTAR

\section{EXPLORING THE HARD X-RAY UNIVERSE}

The Nuclear Spectroscopic Telescope Array (NuSTAR) is a NASA Small Explorer (SMEX) satellite mission that will open the hard X-ray frontier (6-79 keV) to sensitive exploration. NuSTAR is designed to address fundamental questions about our universe outlined in Table 2. Three decades ago, the Einstein mission introduced high-resolution focusing optics to X-ray astronomy. By eliminating high detector backgrounds, allowing true imaging, and permitting the use of compact high-performance detectors, Einstein leapt a factor of a hundred in sensitivity, completely changing our view of the X-ray sky. Now NUSTAR is poised to make the same jump in the high-energy X-ray band, offering a combination of clarity, sensitivity, and spectral resolution surpassing the largest observatories in this band by orders of magnitude.

NuSTAR builds on the High Energy Focusing Telescope (HEFT) balloon program, using a simple design with extensive heritage. The focusing optics described here together with pixelized CdZnTe detectors will make NuSTAR 100 times more sensitive than previous experiments. The NuSTAR mission has been confirmed and having moved to Phase C/D, flight hardware has begun to be built. The NuSTAR launch is schedule for August 2011.

\section{NUSTAR OPTICS DESIGN AND PRODUCTION}

NuSTAR consists of a single instrument that achieves its science objectives with a combination of surveys and pointed observations. The robust mission design is based on a 3-axis stabilized spacecraft and simple operations concept. A Pegasus launch to a $6^{\circ}$ inclination, low-earth (550$600 \mathrm{~km}$ ) orbit provides a stable, low-background environment. A summary of the instrument parameters is given in Table 1 .

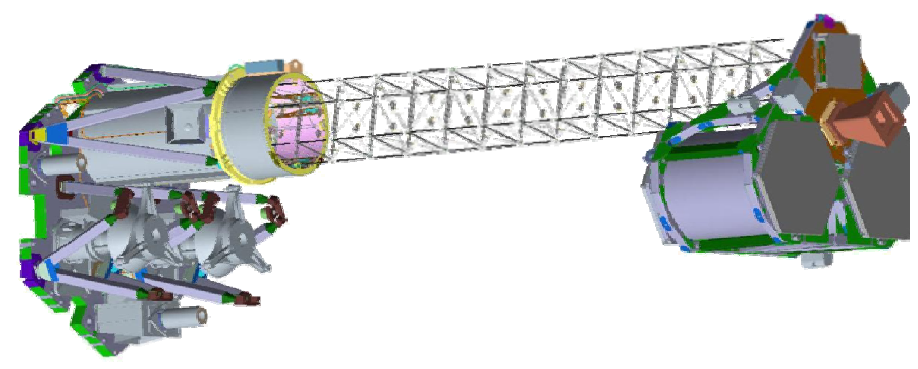

Figure 1: NuSTAR instument (partially deployed).

\footnotetext{
*Correspondence: koglin@astro.columbia.edu, http://www.astro.columbia.edu/ koglin
} 
Table 1: NuSTAR science objectives and preliminary time allocation for the first two years of the baseline NuSTAR mission.

\begin{tabular}{|c|c|c|}
\hline Science Objective & Observation Requirement & $\begin{array}{l}\text { Time Allocated in } \\
\text { Mission }\end{array}$ \\
\hline $\begin{array}{l}\text { Supermassive black hole survey: } \\
\text { How are black holes distributed across the } \\
\text { cosmos, and how do they affect the formation of } \\
\text { galaxies? }\end{array}$ & $\begin{array}{l}\text { Extragalactic surveys: } \\
\text { - Deep survey of } \geq 200 \mathrm{arcmin}^{2} \\
\text { - Shallow survey of } \geq 4 \mathrm{deg}^{2}\end{array}$ & $\begin{array}{l}\text { 10.5 months total: } \\
\text { - } 4 \text { months deep } \\
\text { - } 6.5 \text { months wide }\end{array}$ \\
\hline $\begin{array}{l}\text { Collapsed stellar remnants: } \\
\text { How are stellar remnants distributed within the } \\
\text { Galaxy and near the Galactic center? }\end{array}$ & $\begin{array}{l}\text { Galactic survey(s): } \\
\text { - Survey } \geq 1 \operatorname{deg}^{2} \text { Galactic center }\end{array}$ & 4 months total \\
\hline $\begin{array}{l}\text { Supernova (SN) explosion dynamics and } \\
\text { nucleosynthesis: } \\
\text { How do stars explode and forge the elements that } \\
\text { compose the Earth? }\end{array}$ & $\begin{array}{l}\text { Mapping young SN remnants: } \\
\text { - Cassiopeia A } \\
\text { - SN 1987A }\end{array}$ & $\begin{array}{l}2 \text { months total: } \\
\text { - } 1 \text { months per target }\end{array}$ \\
\hline $\begin{array}{l}\text { Blazars and very high energy sources: } \\
\text { What powers nature's most powerful cosmic } \\
\text { accelerators? }\end{array}$ & $\begin{array}{l}\text { Blazars \& gamma-ray sources: } \\
\text { - Observe } 4 \text { sources, } 3 \text { coordinated } \\
\text { with gamma-ray and optical facilities }\end{array}$ & 1.5 months total \\
\hline $\begin{array}{l}\text { Nearby supernovae: } \\
\text { How do stars explode and forge the elements that } \\
\text { compose the Earth? }\end{array}$ & $\begin{array}{l}\text { Targets of Opportunity (ToOs): } \\
\text { - Observe any SNIa closer than } \\
\text { Virgo or core collapse SN in Local } \\
\text { Group within } 48 \mathrm{hr} \text {. }\end{array}$ & $\begin{array}{l}\text { Part of } 6 \text { month } \\
\text { science reserves }\end{array}$ \\
\hline Science reserves & $\begin{array}{l}\text { Other science objectives: } \\
\text { - Internal proposals by science team }\end{array}$ & 6 months total \\
\hline
\end{tabular}

\subsection{Instrument Overview}

The NUSTAR instrument consists of an array of two co-aligned hard X-ray telescopes. The grazing incidence mirrors focus onto two shielded solid-state pixel detectors, separated by a mast that extends the focal length to 10.15 meters after launch. A laser metrology system monitors the mast alignment. The optics utilize thin glass shells coated with depthgraded multi-layers to extend the bandpass and field of view (FOV) over that achievable with standard metal surfaces. Cadmium Zinc Telluride (CdZnTe) detectors provide excellent spectral resolution and high quantum efficiency without requiring cryogenic operation. ${ }^{1} \quad$ The detectors and their associated electronics are housed in $\mathrm{Pb} / \mathrm{CsI}$ scintillator
Table 2: Instrument Fact Sheet (current best estimates).

\begin{tabular}{|l|l|}
\hline Parameter & Value \\
\hline Energy Range & $6-79 \mathrm{keV}$ \\
\hline Angular Resolution & $46^{\prime \prime}(\mathrm{HPD})$ \\
\hline Field of View & $12 \mathrm{arcmin}$ X 12 arcmin \\
\hline Spectral Resolution & $1.25 \mathrm{keV}$ at $68 \mathrm{keV}$ \\
\hline Timing Resolution & $1 \mathrm{msec}$ \\
\hline ToO response & $<$ few $\mathrm{hr}-24 \mathrm{hour}$ \\
\hline Launch Date & August 15,2011 \\
\hline Orbit & $550 \times 600 \mathrm{~km}, 6^{\circ}$ inclination \\
\hline & $1.8 \mathrm{e}-15 \mathrm{erg} / \mathrm{cm} 2 / \mathrm{s}(6-10 \mathrm{keV})$ \\
Minimum Detectable Flux & $1.7 \mathrm{e}-14 \mathrm{erg} / \mathrm{cm} 2 / \mathrm{s}(10-40 \mathrm{keV})$ \\
(106 $\mathrm{sec}, 50 \%$ extraction) & $1.04 \mathrm{e}-13 \mathrm{erg} / \mathrm{cm} 2 / \mathrm{s}\left(4^{\circ} 10-79\right.$ \\
& $\mathrm{keV})$ \\
\hline
\end{tabular}


shielding. An illustration of the partially deployed instrument is shown in Figure 1 and an overview of the instrument configuration is given in Table 3 .

\subsection{Optics Design}

The two optical modules on NuSTAR collect the incoming hard X-rays and focus them on to the detector. The optical design is a conical-approximation to a Wolter-I, providing high performance across the 12'x12' detector field of view. The optics (c.f., Figure 3 ), are built of thermally formed glass substrates, precisely held in position by epoxy and graphite spacers.

The NuSTAR optics are composed of 133 concentric layers of conical-approximation Wolter I mirrors aligned and held together by epoxy and precisely machined graphite spacers that run along the optic axis. Details of the instrument design are provided in Table 4. Each layer is composed of an upper and a lower set of segmented mirrors, $0.21 \mathrm{~mm}$ thick and $225 \mathrm{~mm}$ long. The innermost (outermost) layer has a radius of 54.4 $\mathrm{mm}(191.2 \mathrm{~mm})$ and a conic graze angle of $1.3 \mathrm{mrad}$ $(4.7 \mathrm{mrad})$. The inner 65 layers are composed of $6 \times$ $\sim 60^{\circ}$ sectors (sextants) and the outer 65 layers are composed of $12 \times \sim 30^{\circ}$ sectors (twelvetant), each aligned with five axial spacers. The transition from sextant to twelvetant mirrors is made with three intermediate sextant layers using nine spacers per sector with wide outside spacers that are shared by adjacent mirrors to provide azimuthal connectivity of the three transition layers. The three transition layers are currently only required for structural support and are not required to provide any effective area. However, the intermediate transition layers are expected to be made of flight-quality mirrors with multilayer coatings and it is estimated that they will provide

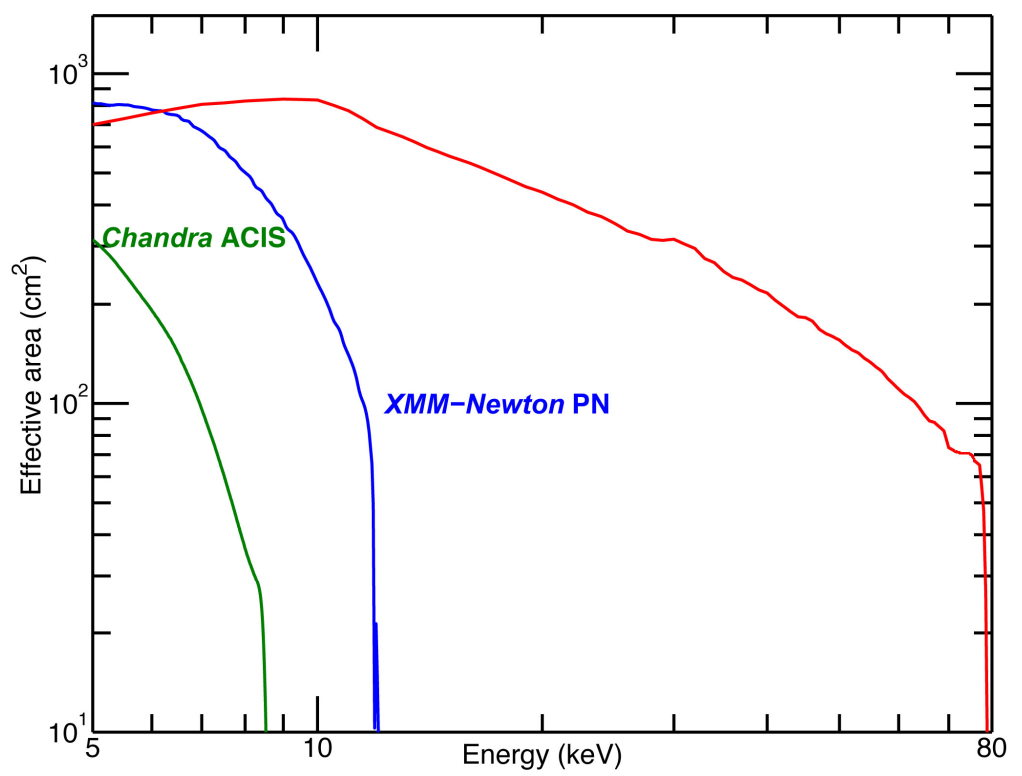

Figure 2: NuSTAR effective area plotted as a function of X-ray energy along with the effective area of other missions.
Table 3: NuSTAR instrument configuration.

\begin{tabular}{|l|l|}
\hline Parameter & Value \\
\hline \# telesc. modules & 2 \\
\hline Eff. focal length & $10.15 \mathrm{~m}$ \\
\hline Optics & Grazing incidence. Conical approx. \\
\hline Reflecting surface & W/Si \&Pt/SiC graded multilayers \\
\hline Sensors & CdZnTe pixel hybrid \\
\hline Shielding & Pb/Csl scintillator \\
\hline
\end{tabular}

Table 4: Telescope parameters

\begin{tabular}{|l|l|}
\hline \multicolumn{2}{|c|}{ Mirror Parameters } \\
\hline Geometry & Segmented conical approx. \\
\hline Substrates & Thermally formed glass \\
\hline Shells/module & $130+3$ transition layers \\
\hline Shell thickness & $0.21 \mathrm{~mm}$ \\
\hline Shell length & $450 \mathrm{~mm}$ \\
\hline Inner radius & $54.4 \mathrm{~mm}$ \\
\hline Outer radius & $191.2 \mathrm{~mm}$ \\
\hline Focal Length & $10.15 \mathrm{~m}$ \\
\hline \multicolumn{3}{|c|}{$\quad$ Multilayer Parameters } \\
\hline Materials & W/Si (outer shells) \\
& Pt/SiC (inner shells) \\
\hline Design & Depth graded \\
\hline Max. thickness & $1.0 \mu \mathrm{m}$ \\
\hline Max. layer pairs & 291 \\
\hline \multicolumn{2}{|r|}{ Focal Plane (FP) Module Parameters } \\
\hline CdZnTe Pixel pitch & $0.605 \mathrm{~mm}$ \\
\hline CZT Units/FP & 4 \\
\hline Pb/Csl shield thick. & $2 \mathrm{~mm} / 1.5 \mathrm{~cm}$ \\
\hline \multicolumn{2}{|c|}{}
\end{tabular}
an additional $\sim 2 \%$ effective area above the baseline calculations described here. Depth-graded multilayers are applied to the optical surfaces in a production coating facility at the Danish Technical University (DTU) that uses planar magnetron sputtering. The multilayers provide coherent reflectivity over a broad energy band.

The entire assembly is built on, and aligned to, a central mandrel. After construction, support spiders are attached to the optic for instrument integration. During fabrication, glass and graphite layers are built outward from the central mandrel and all ground handling and alignment activities use the inner mandrel as a support and reference axis. Once the telescopes are mounted to the telescope bench, each is supported and aligned from attachment points on the spider supports. 


\subsection{Instrument Performance}

NuSTAR will be the first satellite mission to employ focusing optics in the hard X-ray band, extending the reach of current soft X-ray instruments by nearly a decade in energy. The total on-axis effective area for NuSTAR is plotted as a function of energy in Figure 2 and compared to current focusing instruments. The focusing optics of NuSTAR deliver a dramatic improvement in sensitivity over the previous generation of coded aperture and collimated optics used for hard $\mathrm{X}$-rays. The minimum detectable flux for $10^{6}$ second observations in several energy bands is listed in Table 1 .

\subsection{Substrate Production}

Segmented glass optics with multilayer coatings were first introduced for the HEFT balloon mission. ${ }^{2,3,4}$ The HEFT approach of slumping glass microsheets into standard quartz mandrels using commercial ovens to achieve 40" angular resolution after mounting was the original baseline for the NuSTAR mission. ${ }^{5}$ After NuSTAR was restarted in the fall of 2007, GSFC joined the collaboration to build the flight mirrors. The GSFC approach is to form the substrates onto polished forming mandrels. ${ }^{6}$ The figure of the glass substrates is measured with an interferometer (GSFC) and/or a laser scanning system (Columbia). At least $10 \%$ of the mirrors are measured with the interferometer immediately after forming for quality assurance and feedback into the forming process.

Multilayer coatings are required to enhance hard X-ray $(>10 \mathrm{keV})$ reflectivity with a broad energy acceptance. A multilayer structure is a stack of thin layers of alternating materials designed so that the small reflections from each layer add in phase. Depth-graded multilayers vary the bi-layer thickness so that different layers are optimized to reflect different wavelengths, providing broadband response. Depth-graded multilayers are applied to the optical surfaces in a production coating facility at DTU that uses planar magnetron sputtering. The specific multilayer design depends on graze angle, which scales with the optic radius. The 130 shells (plus three transition shells) are divided into 10 groups optimized for a particular range of graze angles. ${ }^{7}$

Before coating, the substrates are cleaned using a three step ultrasonic process and sorted by radius for application of the appropriate design. The DTU facility can coat $0.8 \mathrm{~m}^{2}$ in substrate surface area in a single run (pump-down, coating and venting of the chamber); each run is a one-day operation. Producing the required number of substrates consistent with the NuSTAR schedule requires coating $1.9 \mathrm{~m} /$ week, compared to the $4 \mathrm{~m}^{2} /$ week capability, providing significant margin. Quality is assured for each coating run by including a silicon wafer as a witness that is characterized at $8 \mathrm{keV}$ for reflectance spectra. These reflectance data, as well as housekeeping data from the coating chamber are automatically logged to the optics database, also updated to reflect the coating applied to each substrate. The material combinations for the coatings depend on graze angle - the inner 7 radius groups (about $2 / 3$ of the geometric area) are coated with $\mathrm{Pt} / \mathrm{SiC}$, and the remaining are coated with $\mathrm{W} / \mathrm{Si}$. Details of the multilayer design and optimization is described elsewhere. ${ }^{7}$ After multilayer coating, the figure of each mirror is measured with the laser scanner at Columbia before mounting.

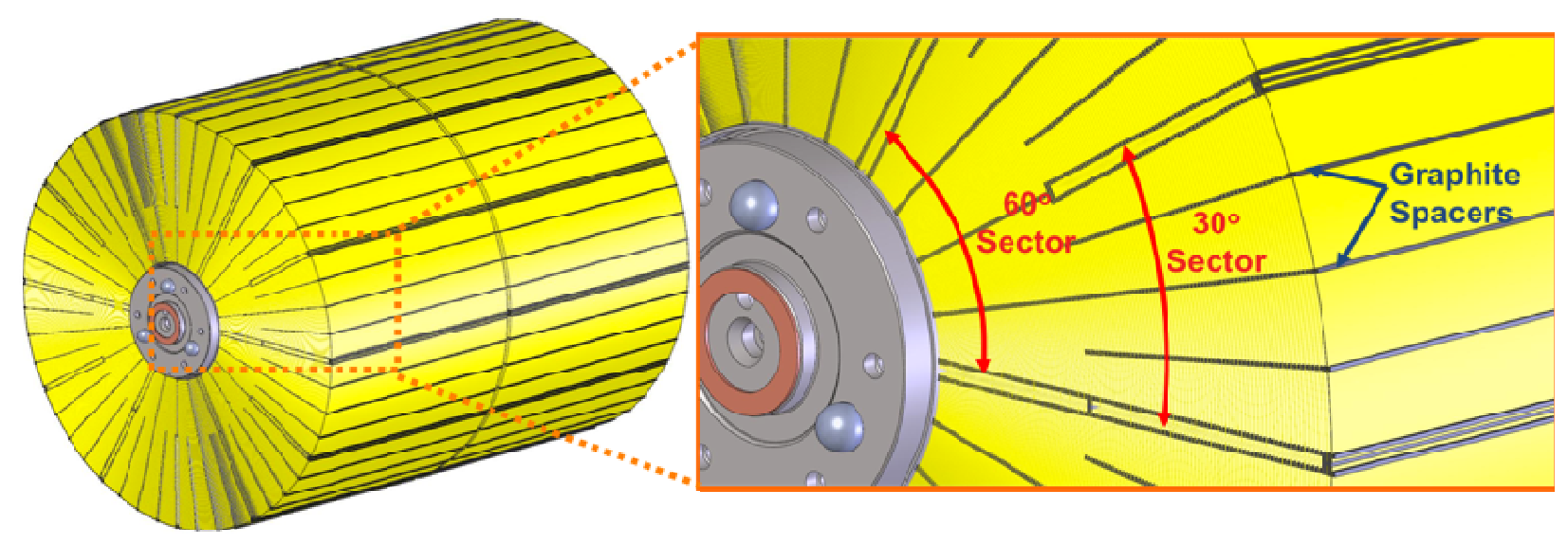

Figure 3: The NuSTAR optics are composed of 133 concentric layers of conic-approximation Wolter I mirrors aligned and held together by epoxy and precisely machined graphite spacers that run along the optic axis. The inner 65 layers are comprised of 6 $\times \sim 60^{\circ}$ sectors (sextants) and the outer 65 layers are comprised of $12 \times \sim 30^{\circ}$ sectors (twelvetant), each aligned with five axial spacers. The transition from sextant to twelvetant mirrors is made with three intermediate sextant layers using nine spacers per sector with wide outside spacers that are shared by adjacent mirrors to provide azimuthal connectivity between adjacent layers. 


\subsection{Optics Assembly}

The coated substrates are assembled into an optic using two new assembly machines at Columbia University's Nevis Laboratory. The assembly machines, which were built by $\mathrm{ABtech}^{\dagger}$, comprise precision air-bearing axes mounted on solid granite bases and provide exceptional machining tolerances. The machines are housed in a class 1000 clean room with temperature and humidity control, and the air-bearings machine components require no oils that could potentially contaminate the optics. Our error-compensating, monolithic assembly and alignment (EMAAL) procedure involves constraining the mirror segments to precisely machined graphite spacers that run along the optical axis. In this process, the nominally cylindrical glass segments are forced to a conical form, and in the process, radial mismatches are removed. In order to achieve large effective area, concentric layers of glass are stacked on top of each other starting with a central mandrel. Graphite spacers are first epoxied to the mandrel and then precisely machined to the correct radius and angle. Next, a layer of glass and second layer of spacers are epoxied to the first set of spacers. These spacers are then machined to the appropriate radius and angle. This process is repeated until the requisite number of layers is assembled. A key point of this process is that each layer of spacers is machined with respect to the optic axis and not the last layer of glass. In this way, there is never any stack-up error during the telescope fabrication. Each layer requires one workday to complete. The two machines will operate in parallel, each producing one layer per day such that each optics module will be completed in $\sim 6$ months. Since the schedule allows only a limited time for optics calibration, a third test and calibration optic will be built and evaluated through a more extensive calibration procedure.

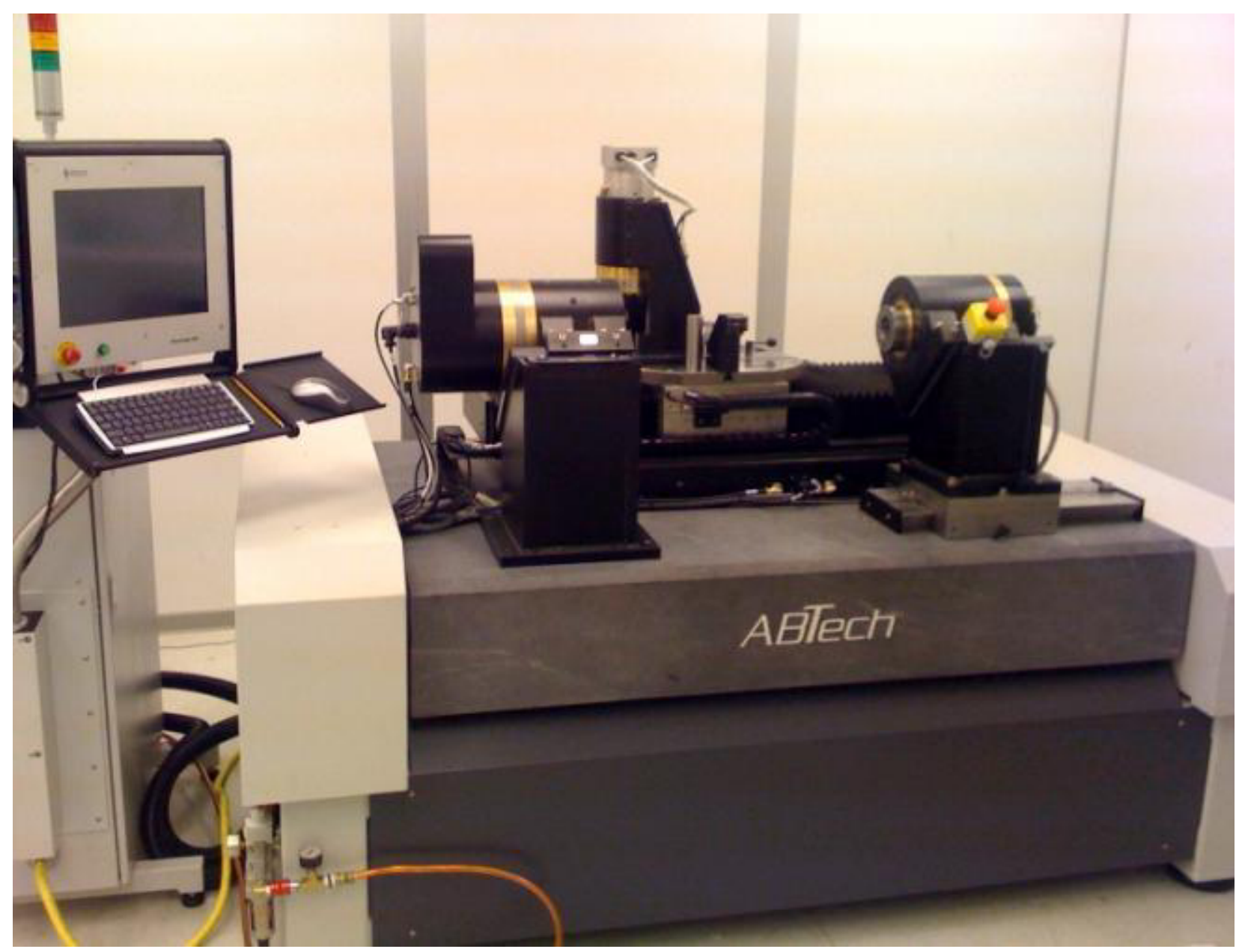

Figure 4: NuSTAR assembly machine installed inside a class 1000 clean room at Columbia University's Nevis Laboratory.

\footnotetext{
$\dagger^{\dagger}$ http://www.abtechmfg.com
} 


\section{NUSTAR OPTICS PERFORMANCE}

The NuSTAR optics performance requirements are driven by the science goals. For background limited observation, the minimum detectable flux sensitivity (c.f., Table 1) goes as

$$
F_{\text {min }} \propto \frac{\theta_{P S F}}{A_{\text {eff }}} \sqrt{\frac{B_{\mathrm{det}}}{t}},
$$

where $\theta_{P S F}$ is the instrument point spread function (PSF), $A_{\text {eff }}$ is the effective area, $B_{\mathrm{det}}$ is the detector background count rate, and $t$ is the observation time. In addition to being critical to the instrument sensitivity, the width of the PSF is also key to resolving extended sources as well as limiting potential source confusion. The optics contribute the dominant portion of the overall instrument PSF with a small additional contribution from pointing error and the detector pixilation (CdZnTe pixel size $=0.605 \mathrm{~mm}$ or $12 ")$. The detector has high efficiency over the entire energy range and there are only small losses at low energy due to the optics thermal covers and a thin window at the focal plane (i.e., > $90 \%$ combined efficiency over the entire energy band). In this section we describe the optics contribution to the effective area and PSF.

\subsection{Point Spread Response Function}

The optics performance dominates the instrument PSF with some additional contribution from aspect reconstruction and detector spatial resolution. The optics contribution to the instrument PSF is broken up into three categories: initial figure of the thermally formed substrates, performance after mirror alignment and assembly, and performance on-orbit including the thermal operating environment. The initial figure error of the conical substrates is required to have an average of 30" HPD. A more detailed description of the NUSTAR substrate production and performance is given elsewhere. ${ }^{6}$ The optics performance after mounting and alignment is specified to be 39" HPD with an on-orbit specification of 43" HPD. The uniformity and stability of the epoxy bonds is crucial to meeting and maintaining these performance specifications, and details of the epoxy selection and performance are given elsewhere. ${ }^{8}$ The On-axis PSF is radially symmetric. For sources increasing further off-axis, the 2D PSF will become less symmetrical (i.e., more bowtie like than circular), but the HPD is not expected to change significantly.

Our ability to meet the NuSTAR optics PSF requirement of 43" HPD (half power diameter) was first reported over five years ago. ${ }^{5,9}$ Since then numerous prototypes consisting of a few segments to multiple layers have been built to investigate specific aspects of the optics design and assembly process. This includes detailed study of the spacer width and configuration; different epoxy types and application methods; machining techniques; mounting hardware; and substrates and mulitilayer coatings. As an example, a recent prototype composed of 10 uncoated mirror segments mounted in a conical configuration was found to have an HPD of 45" (see Figure 5). The PSF results were generated using a raytrace based on axial surface height measurements (LVDT scans) performed every 2.5 in azimuth. In the past this procedure has been demonstrated to agree well with subsequent X-ray pencil beam scan measurements performed at $8 \mathrm{keV} ., 3,5,9,10$ The mirrors had NuSTAR flight quality performance (average freestanding performance of 30") and near flight representative geometry (sextant geometry, $0.21 \mathrm{~mm}$ thick, and $200 \mathrm{~mm}$ long instead of the $225 \mathrm{~mm}$ long NuSTAR prescription). From the freestanding performance we deduce a $\sim 30$ " mounting contribution to the total mounted HPD. This prototype was built using the old assembly machine, which was used to build the HEFT telescopes, as well as old mounting hardware. The improved dust/contamination control of the new assembly machines along with improved mounting hardware is expected to provide improved mounting performance.

\subsection{Effective Area}

The NuSTAR effective area has been calculated using two separate raytrace codes with good agreement. The input assumptions include 43" HPD and the optimized multitilayer recipes detailed in Madsen et al. ${ }^{7}$ The calculated effective area is plotted as a function of energy in Figure 6 for source positions up to 9' off-axis in 1' steps. The FOV defined as the off-axis position for which the effective area has dropped by a factor of $50 \%$ relative to the on-axis effective area ranges from a diameter of $13^{\prime}$ at the lowest energies (i.e., a radius of $6.5^{\prime}$, which basically covers the entire focal plane detector) to a diameter of $6^{\prime}$ at the highest energies. The decrease in effective FOV at higher energies is due to a steeper decrease in reflectivity as a function of graze angle at higher energy. Further, the optics vignetting function increases with shell diameter and since the lower energy X-rays have relatively more effective area in the outer shells than the higher energy X-rays, the low energy X-rays experience less off-axis vignetting overall. As previously mentioned, the effective area calculations do not include any contribution from the three intermediate transition layers, which are expected to provide an additional $\sim 2 \%$ effective area over the entire energy band. 


\section{SUMMARY}

As the first satellite mission to employ focusing optics in the hard X-ray band, NuSTAR is poised to usher in a new period of discovery. In this paper, we have presented an overview of the NuSTAR optics design, production and performance.

\section{ACKNOWLEDGEMENTS}

This work is supported by a NASA contract to Columbia University, NNG08FD60C, "The Nuclear Spectroscopy Telescope Array (NuSTAR): Bringing the High Energy Universe into Focus.”
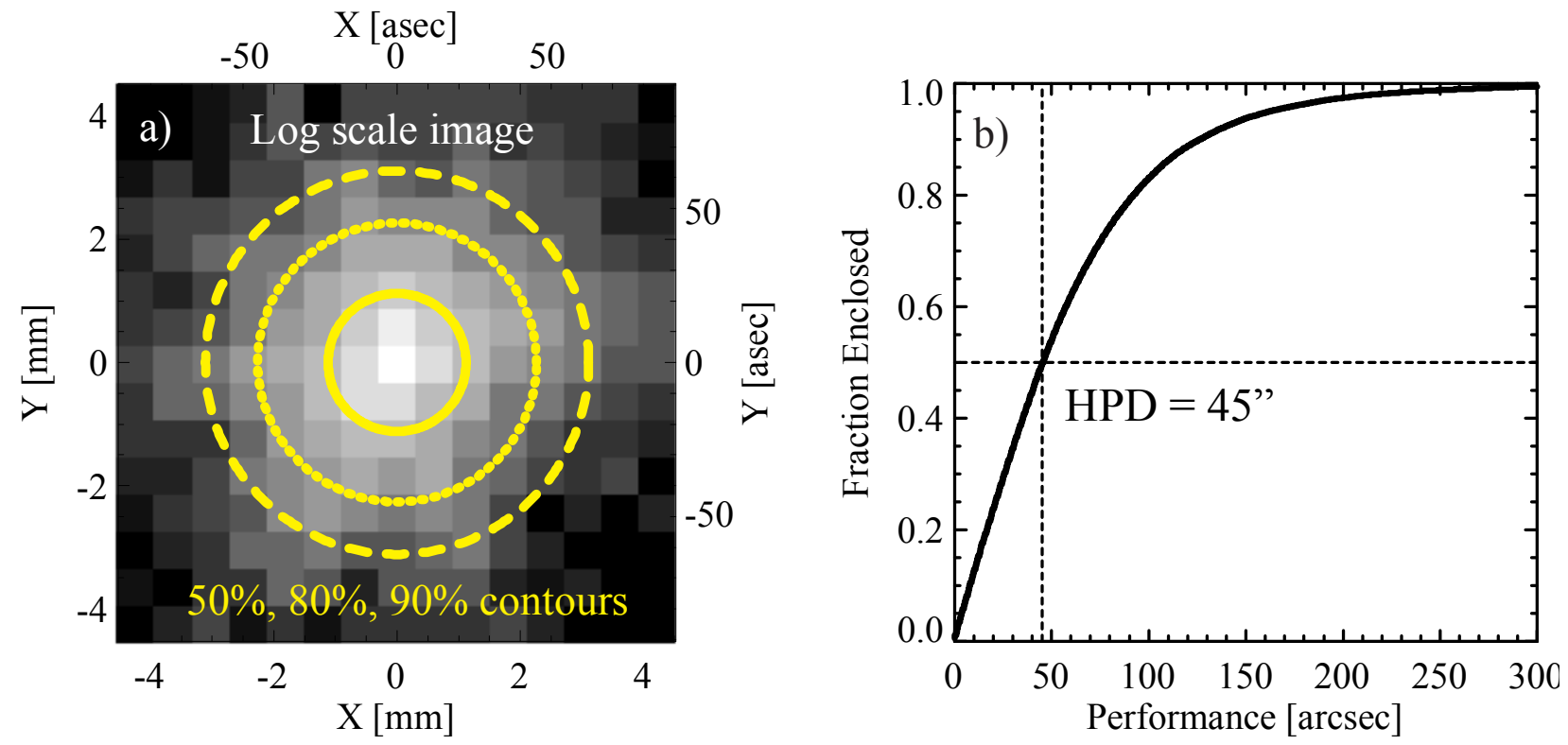

Figure 5: a) 2D reconstructed image from LVDT surface height measurements with log intensity scale of recent prototype consisting of 10 individual mirrors mounted in a conic mirror configuration. The pixel squares of $0.6 \mathrm{~mm}$ are the size of the NuSTAR CdZnTe detectors. The 50\%, 80\% and 90\% encircled energy contours are plotted with solid, dotted and dashed lines respectively. b) Plot of the fraction of energy enclosed versus performance diameter.
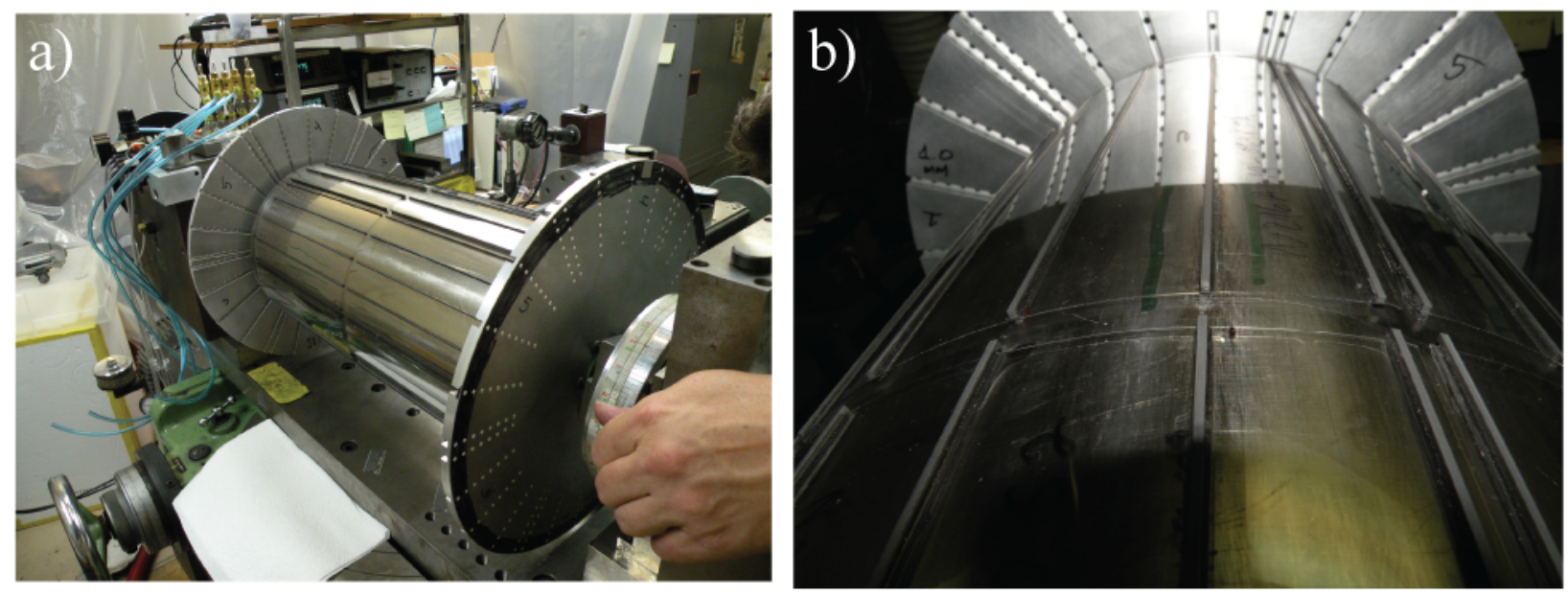

Figure 6: a) Image of recent prototype optic being built on old assembly machine used for HEFT and NuSTAR development work.

b) Closeup image of optic showing lower segment and part of the upper segment along with the graphite spacers that run along the optical axis that are used to mount and align the cylindrical shells to their correct conic shape. 


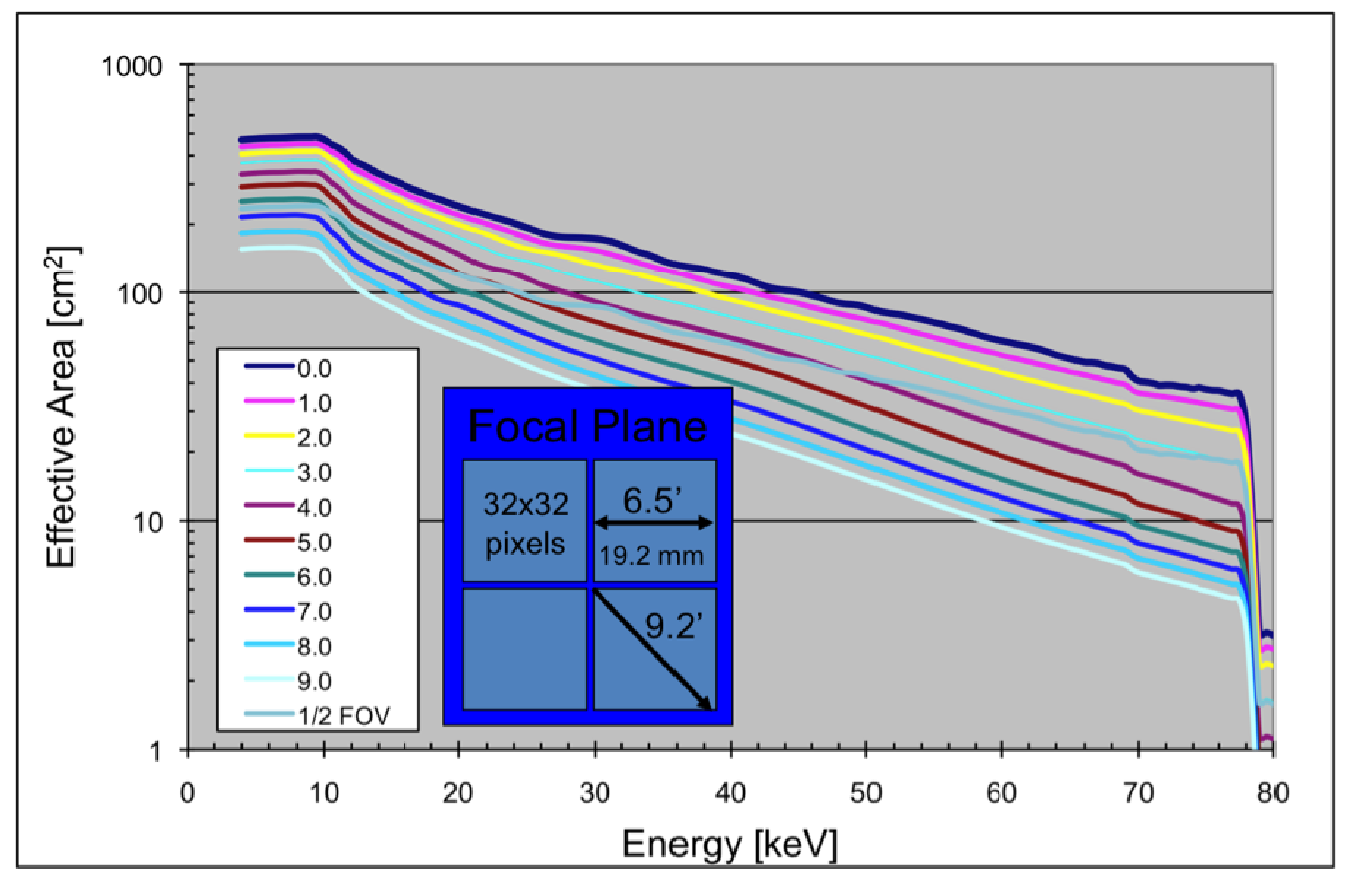

Figure 7: The effective area for a single optic is plotted versus energy for source positions ranging up to 9' off-axis. The FOV defined as the off-axis position for which the effective area has dropped by a factor of $50 \%$ relative to the on-axis effective area is also plotted as a function of energy and ranges from a diameter of 13' at the lowest energies (i.e., a radius of 6.5' which basically covers the entire focal plane detector) to a diameter of 6' at the highest energies. An inset illustration of the four hybrid CdZnTe detectors that make up the focal plane shows the relative size of the focal plane.

\section{REFERENCES}

${ }^{1}$ V. Rene et al., "Performance of the NuSTAR focal plane detectors", Proc. SPIE 7435-2 (2009).

${ }^{2}$ J.E. Koglin, F.E. Christensen, J. Chonko, W.W. Craig, T.A. Decker, C.J. Hailey, F.A. Harrison, C.P. Jensen, M. Sileo, D.L. Windt and H. Yu, " Development and Production of Hard X-ray Multilayer Optics for HEFT," Proc. SPIE 4851, 607-618 (2003).

${ }^{3}$ J.E. Koglin, H.C. Chen, F.E. Christensen, J. Chonko, W.W. Craig, T.R. Decker, M.A.Jimenez-Garate, C.J. Hailey, F.A. Harrison, C.P. Jensen, M. Sileo, D.L. Windt and H. Yu, "Development of Precision Hard X-ray Multilayer Optics with Sub-Arcminute Performance," Proc. SPIE 4851, 673-683 (2003).

${ }^{4}$ J.E. Koglin, C.M.H. Chen, F.E. Christensen, J. Chonko, W.W. Craig, T.R. Decker, K. S. Gunderson, C.J. Hailey, F.A. Harrison, C.P. Jensen, K.M. Madsen, M. Stern, D.L. Windt and H. Yu, E. Ziegler, "Production and calibration of the first HEFT hard X-ray optics module", Proc. SPIE 5168, 100-111 (2004).

${ }^{5}$ J.E. Koglin, C.M.H. Chen, J.C. Chonko, F.E. Christensen, W.W. Craig, T.R. Decker, C.J. Hailey, F.A. Harrison, C.P. Jensen, K.K. Madsen, M.J. Pivovaroff, M. Stern, D.L. Windt, E. Ziegler, "Hard X-ray Optics: From HEFT to NuSTAR", Proc. SPIE 5488, 856 (2004).

${ }^{6}$ W.W. Zhang, "Manufacture of mirror substrates for the NuSTAR mission”, Proc. SPIE 7437-20 (2009).

${ }^{7}$ K.K. Madsen et al., "Optimizations of $\mathrm{Pt} / \mathrm{SiC}$ and W/Si multilayers for the Nuclear Spectroscopic Telescope Array", Proc. SPIE 7437-37 (2009).

${ }^{8}$ H. An et al., "Contamination modeling and epoxy selection and evaluation for the NuSTAR hard X-ray optics", Proc. SPIE 7437-48 (2009).

${ }^{9}$ J.E. Koglin et al., "NuSTAR Hard X-ray Optics”, Proc. SPIE 5900-33 (2005).

${ }^{10}$ J.K. Koglin et al., "Calibration of HEFT Hard X-ray Optics”, Proceedings of The X-ray Universe 2005, 955 (2006). 